\title{
How to Improve the Laparoscopic Technique of Urologist
}

\author{
Xuezhen Yang*, Jie Fu and Xueping Ma \\ Department of Urology, The Second Affiliated Hospital of Bengbu Medical College, China
}

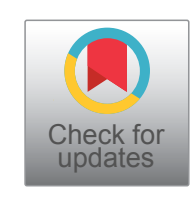

Since the 1990s, the rapid development of laparoscopic technology has led urologists into a new era of Urology - minimally invasive era. Compared with open surgery, laparoscopic surgery has the advantages of less surgical injury, clear surgical field and anatomical structure, rapid postoperative recovery, less perioperative complications, beautiful incision and short average hospital stay. It has become the mainstream of urological surgery. At present, almost all urological surgery could be completed by laparoscopy. Worldwide, in major medical centers, the proportion of minimally invasive surgery has exceeded that of open surgery. Therefore, laparoscopic technology is a necessary skill to learn and master in the growth of urologists.

Any excellent laparoscopic surgery, even if the operation is difficult and the steps are cumbersome, is also composed of countless basic laparoscopic operation technologies. If each step could be done well and in place, the whole operation could be carried out smoothly and give visitors a beautiful enjoyment. Similarly, practicing the basic skills of laparoscopic surgery could shorten the learning curve of laparoscopic surgery and complete more complex and difficult operations for urologists. For patients, it could reduce the operation and anesthesia time and reduce perioperative complications.

The basic skills of laparoscopic surgery and open surgery are similar but different. The basic operations of laparoscopic surgery and open surgery include separation, hemostasis and suture. Open surgery is performed under the 3D effect of human eyes, laparoscopic surgery is performed on a 2D display screen. Open surgical instruments are short and easy to use and master, laparoscopic instruments are long, difficult to operate, and carried out under the plane display effect, which requires more practice. In addition, the assistant of open surgery is mainly to help the chief surgeon to pull and expose the surgical area, which is equivalent to the additional hand of the chief surgeon. The transition from assistant to chief surgeon is relatively easy, the assistant of laparoscopic surgery needs to start with the "mirror hand", which is equivalent to the eyes of the surgeon in charge, and then gradually transition to laparoscopic operation. The transition from assistant to surgeon in charge requires more assiduous simulation training.

\section{How to Do Laparoscopic Simulation}

\section{Production of simulator laparoscopic surgery has a certain learning curve as open surgery}

Open surgery could be trained step by step in the process of surgery. Young doctors could separate non key steps from opening and closing incision to auxiliary surgeon, and then gradually transition to key steps of surgery. Most operations under laparoscopy are completed by the chief surgeon, and the assistant could rarely get the opportunity of practical operation. The lack of practical training for laparoscopic operation leads to the extension of learning curve. Basic skills of laparoscopic surgery training often start with an in vitro simulator and then gradually transitions to in vivo operation. Therefore, how to obtain a suitable simulator is very important for novices in laparoscopic surgery.

There are many finished products for sale of laparoscopic simulators, but self-made simple simulators are recommended in terms of economy and practicability. First, we could understand the intraperitoneal structure through a self-made simulator. Second, we could choose different materials to obtain simulation training in different positions, angles and spaces. The materials needed to make the laparoscopic simulator are as follows: 1) Camera (high definition, self-contained LED lamp, adjustable angle); 2) Carton (recommended external box of medical saline); 3) Laptop (or tablet computer). After the carton is closed, the opening is used as the operation instrument channel, and the computer is connected to the camera to start the in vitro simulation training of laparoscopy. If the opening on the carton is inappropriate or to simulate different scenes under laparoscopy, the carton could be replaced by a new one.

*Corresponding author: Xuezhen Yang Department of Urology, The Second Affiliated Hospital of Bengbu Medical College, Bengbu, Anhui, 233020, China

Accepted: February 05, 2022

Published online: February 07, 2022

Citation: Yang X, Fu J, Ma X (2022) How to Improve the Laparoscopic Technique of Urologist. Adv Laparoscopy 5(1):127130

Copyright: (C) 2022 Yang X, et al. This is an open-access article distributed under the terms of the Creative Commons Attribution License, which permits unrestricted use, distribution, and reproduction in any medium, provided the original author and source are credited. 


\section{Selection of laparoscopic instruments for in vitro simulation training}

It is recommended to purchase the same surgical instruments as those in daily surgery, so as to achieve the hand feeling and intracavitary operation feeling related to surgery. Don't buy cheap and inferior training equipment to save money. There are not many instruments required for laparoscopic simulation training. A pair of separation forceps, flat head forceps, scissors and needle holders could complete most of the simulation operations. Some stitches and sutures should also be prepared for laparoscopic suture simulation.

Contents of simulator operation training the contents of simulator operation training are diverse, and it is recommended to carry out it step by step from simple to complex. Start with the simplest laparoscopic spatial sense and clamping training. At this time, you could take the training method of sandwiching beans or corn from one small bowl to another. Next, you could find some rubber hair rings, clamp them with your left hand, transfer them to your right hand, and then trap them on the prepared nails or piles. Then you could train yourself independently and cutting skills could be trained by peeling grapes or paper cutting. The training is to put the grapes into the simulator, separate the pliers and scissors with your left hand, and peel the grape skin from the grapes, draw a pattern on the paper in advance and cut it off with separating pliers and scissors. In this way, the techniques of left and right hand cooperation and separation and cutting could be trained. You could then further practice stitching and knotting techniques. It is to find a piece of silica gel or pork, hold it with a left-hand separating forceps and a righthand needle, sew it continuously or intermittently, and then tie a knot. When the above in vitro simulation operation has been practiced skillfully, it could be practiced pertinently. For example, if you want to train partial nephrectomy, you could buy a complete pig kidney, mark the position to be removed in advance, fix it in the simulator according to the angle of operation, remove part of the renal parenchyma and suture.

Extracorporeal operation training is always boring, but it is an indispensable part of laparoscopic surgery. It is suggested that timing should be carried out at the same time of extracorporeal operation training to calculate the number of effective operations and growth curve per unit time. At the same time, timing could also increase the pressure during training and better simulate the operation of real surgery.

\section{How to Practice the Basic Technique of Laparoscopy}

\section{Separation is for better exposure, and good exposure is the premise of successful operation}

Laparoscopic exposure mainly depends on the operator's left hand instruments and assistant instruments. The extraperitoneal approach usually used in urology could rely on gas to open the peritoneum and intra-abdominal organs, reduce auxiliary trocar compared with transabdominal surgery, avoid damaging the peritoneum as much as possible, fully free and expand the operation space. If the exposure is not good and the visual field is not clear, it is easy to cause accidental injury, and it is relatively difficult to stop bleeding, which increases the difficulty of operation.

The three basic operations of incision, separation and exposure are combined and inseparable in laparoscopic surgery. The cooperation between the left and right hands of the operator is very important, and the left hand is his "one help". A perfect operation is closely related to the utilization of the operator's left hand.

\section{Hemostasis bleeding in laparoscopic surgery should focus on prevention}

Specific hemostatic measures include electrocoagulation hemostasis, clamping hemostasis and suture hemostasis. Different hemostatic techniques need to be used in combination with the actual situation during the operation, which requires the operator to accurately master each hemostatic method. Only by strengthening the basic skills of hemostasis could we deal with emergencies calmly in the face of danger.

Accurate ligation of blood vessels is the basis of surgical safety. Ligation instruments include Hem-o-lok, absorbable clip, titanium clip, cutting suture device, etc. Before using this kind of instrument, read the instructions and precautions carefully, and master the correct use of ligation instrument. During ligation, it is necessary to fully expose, ensure crossing, avoid clamping into other tissues, avoid being close to the root of blood vessels, and keep away from the proximal ligation clamp as far as possible.

The key to hemostasis lies in the exposure of bleeding points. When the artery is ruptured and bleeding, do not clamp it in a hurry to avoid tearing the wound or causing side injury. You could try to press gauze to fill it. If necessary, open the venous access for blood transfusion. After the preparation is done, slowly remove the gauze to stabilize hemostasis. It could be switched to open or the proximal end of the artery could be clamped with blocking forceps, and the bleeding point could be sutured with vascular suture. In case of venous bleeding, the bleeding is not turbulent due to the existence of pneumoperitoneum pressure under endoscopy. At this time, the empty space of suction device shall be reduced as much as possible. Suction leads to decreased pressure in the cavity and aggravates bleeding. After finding the bleeding point, press the mouth of the right hand suction device at the bleeding point. After the left hand clamp controls the bleeding point, use bipolar electrocoagulation or Hem-o-lok clamp. Small vein breaks could be clamped with golden Hemo-lok or titanium clip for hemostasis, large vein breaks could be clamped continuously with large Hem-o-lok or titanium clip, or sutured with 4-0 Prolene suture.

\section{Skilled suture technique is inseparable from long-term, repeated and standardized training}

To achieve skilled suture, we must master a series of unique laparoscopic suture techniques suitable for different situations through sufficient in vitro simulation training. At the same time, we should often participate in the actual 
operation of laparoscopic surgery, carry out a lot of training practice, and consult and communicate with skilled superior doctors, so as to realize the gradual maturity of suture technology.

Picking up and adjusting needles is the basis of suture. Try to master them on the simulator before operation. In the view of laparoscopy, it is not easy to rely solely on endoscopic instruments for the treatment of suture needles because of the lack of direct assistance of hands. The use of the righthand needle holder needs to be very flexible. The direction of the needle tip could be adjusted through the change of the angle of the needle holder, so as to carry out accurate suture. Needle adjustment could be divided into "three-step", "twostep" and "one-step": "three-step" refers to picking up the needle with the left-hand separating pliers, holding the thread with the right-hand needle, adjusting the needle into a "smiling face" or "crying face" shape, and holding the needle with the right-hand needle and then clamping the needle. "Two step method" means that the right hand picks up the needle directly, and the left hand separating forceps assist in adjusting the needle angle and clamping two needles; "One step" means that when picking up the needle with the right hand, the angle of the needle is directly adjusted by relying on the surrounding unimportant tissue. In addition, from the actual operation of the operation, it is also necessary to train the aspirator to adjust the needle, so as to apply it to suture hemostasis during bleeding, especially in partial nephrectomy.

The position and angle of the clip are also critical. When the needle is held close to the end of the needle, the penetration depth and distance of the tissue could be increased, such as when suturing the wound in laparoscopic partial nephrectomy. When the needle is clamped near the center of the needle, the position and direction of the needle could be accurately controlled, such as laparoscopic anastomosis. It should be emphasized that the tip of the needle holder should be used when holding the needle, so that the angle and direction of the needle could be controlled more flexibly. Do not clamp the needle at the root of the needle holder, first, the needle holder is easy to be damaged, and second, the direction controllability is poor.

\section{How to Use Ultrasonic Knife Well}

Ultrasonic scalpel is the most commonly used operating instrument for urological laparoscopy. The use techniques of ultrasonic scalpel include the following basic actions: 1) Cutting the use of ultrasonic scalpel could cut and separate tissues to ensure accurate hemostasis. Sharp separation should be used as far as possible during separation, so as to reduce bleeding and maintain a clear surgical field. For some thinner blood vessels, the ultrasound knife could be used to disconnect the blood vessels directly. For some thicker blood vessels, they should be located in the middle and front of the ultrasound knife, and the slow coagulation function should be used to coagulate and cut off both sides of the position for a few seconds clock, and then cut it in the middle. 2) Delimitation Similar to the use of electric knife, the non vascular area could be delimited with ultrasonic knife, such as opening the perirenal fascia and separating the ventral and dorsal sides of perirenal fat sac. 3) Support The design of the new generation ultrasonic knife head is more exquisite, which could be used as a separation forceps for fine separation. After the opening of the ultrasonic knife is closed, it is inserted into the vascular sheath or behind the blood vessels, and then opened to assist in the separation of blood vessels. 4) Pulling When dealing with blood vessels, close the knife head, feel the force feedback of the tissue, master the strength, and effectively pull out the tissue around the blood vessels along the direction of the blood vessels to expose the blood vessels. The action range of "dial" is small, and the strength should be gentle. 5) Push For loose tissue space, such as the space between renal fat sac and perirenal fascia, ultrasonic knife and separation forceps could be used for blunt separation. Compared with "dial", the action range of push is larger. When pushing, pay attention to the tactile sensation and force feedback of the organization, and master the direction, speed and force of pushing. 6) Screwing for the tough tissue without blood vessels, the ultrasonic knife could be used to clamp the tissue and turn the knife head to cut while doing work. The purpose is to increase the tension and speed up the cutting speed, such as cutting perirenal fat.

Theoretically, the ultrasonic scalpel could accurately close and cut the vessels with a maximum diameter of $7 \mathrm{~mm}$. In clinical practice, the ultrasonic scalpel could accurately close the adrenal artery, the second lumbar vein on the surface of the left renal artery, and the blood vessels at the junction of bladder and prostate, which provides a lot of convenience for clinical work.

\section{How to Do a Good Job of the Mirror Holder}

The assistant holding the mirror in laparoscopic surgery is equivalent to the operator's eyes and the photographer shooting a film. Becoming a good mirror holder is the only way to grow into an excellent surgeon.

A good mirror holder should pay attention to the following links: 1) Master the direction of laparoscopy Common laparoscopic lenses include $0^{\circ}$ mirror and $30^{\circ}$ mirror. The $0^{\circ}$ mirror is simple to use, but the field of vision is small, and the angle could not be changed. It could only adjust the observation direction. Now it is less and less used. The 30 o mirror has a wide field of vision, and the observation angle of the lens could be adjusted according to different surgical sites, which also has higher requirements for the mirror holder. When holding the $30^{\circ}$ mirror, the direction of the optical fiber should be adjusted according to the different observation parts, and the angle of the mirror body should be changed. In theory, any part of the operation could be observed. When adjusting the fiber direction, make sure the mirror body is always in the positive position (that is, the left and right instruments of the operator are located on the left and right sides of the mirror respectively). 2) Focus on the center of the surgical field of vision the field of vision of laparoscopic surgery should be placed in the center of the screen, just like the central composition method in photography. The focus of the operation should be placed in the center of the screen for composition, so that the main body is prominent and clear, 
and it is easy to achieve the effect of left-right balance. For laparoscopic surgery, the center of gravity is the operating instrument on the right hand of the main knife. For example, the ultrasonic knife and scissors are placed at the focus during separation operation, and the suture needle is placed at the center of the screen during suture. It bring beauty to the operator and the surgical audience. 3) Master the distance of laparoscopy Try to avoid close-up in the field of vision. If there is a close-up view in the field of vision, the laparoscopic focusing and built-in light could not be concentrated in the operation area, which will affect the aesthetic feeling of surgical viewing. Just like in photography, if there is a close range, it will affect the expression of the focused object. In addition, the distance of the mirror body should be adjusted according to the operator's operation. For example, when the operator changes the instrument or sutures to find the needle, the mirror body should be retreated to observe the panorama, so as to quickly locate the position of the replaced instrument or needle. 4) Familiar with the operation process $A$ good mirror holder should not only pay attention to the ongoing operation of the operator and provide the operator with a good field of vision, but also understand the operator's next operation, so as to timely adjust the direction of laparoscopy and ensure the smooth operation [1-3].

In short, any surgery and operation, including laparoscopic surgery, need to be diligent in practice. The so-called "one minute on the stage, ten years off the stage", only by studying and practicing hard off the stage and observing the operation more, could we be comfortable on the stage, shorten the learning curve of laparoscopic surgery, and finally complete the transformation from "imitation and proficiency" to "transcendence and innovation", so as to become a master of minimally invasive surgery.

\section{References}

1. Kimura T, Kojo K, Shiga M, et al. (2021) Impact of early exposure to simulation program on undergraduate medical students' interest in urology. J Med Educ Curric Dev 8: 23821205211020750.

2. Sánchez Fernández J, Bachiller Burgos J, Serrano Pascual A, et al. (2018) Evaluation of competencies in urology. Evaluation of competencies in urology. Arch Esp Urol 71: 73-84.

3. Clements MB, Morrison KY, Schenkman NS (2016) Evaluation of laparoscopic curricula in american urology residency training: $A$ 5-Year Update. J Endourol 30: 347-353.

DOI: $10.36959 / 367 / 454$ 